\title{
Diamond Window Diagnostics for Nuclear Fusion Applications - Early Concepts
}

\author{
F. Mazzocchi, G. Aiello, A. Meier, S. Schreck, P. Spaeh, D. Strauss, and T. Scherer \\ Karlsruhe Institute of Technology, Institut für Angewandte Materialen - Angewandte Werkstoffphysik (IAM-AWP),
}

Association KIT-EURATOM, P.O. Box 3640, D-76021 Karlsruhe, Germany

\section{Introduction}

The main problem, that has so far risen in the field of thermonuclear fusion, regards the plasma instability and the consequent disruptions that occur afterwards. This leads to pulse interruption and reaction extinguishment. The next generation power plants will therefore require an Electron $\underline{\text { Cyclotron }} \underline{\text { Heating and }}$ Current $\underline{\text { Drive system }}$ (ECH\&CD), capable of injecting high power microwave beams where properly needed, in order to destroy magnetic islands and instabilities inside the vacuum vessel. Such a system must be sealed to maintain the vacuum inside the vessel and to act as a barrier for tritium present during pulse operation. A nearly perfect transmission of the microwaves beams used by the ECH\&CD system is mandatory: at MW power levels, even the smallest back reflections might severely damage the gyrotrons, which are responsible for the beam

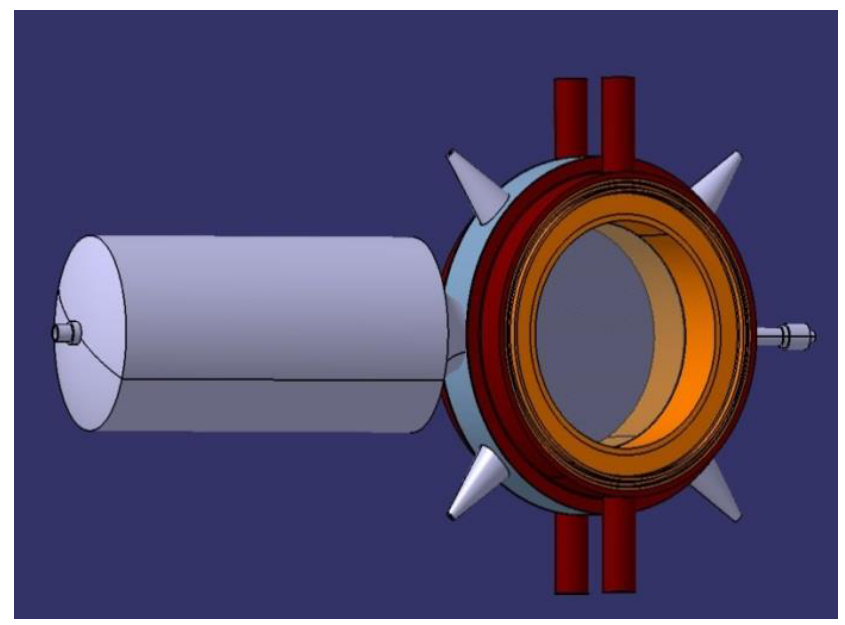

1 Conceptual CATIA model of a diamond window unit, complete with additional diagnostics mock-ups

generation. Due to the power levels of the beams and the stress they must endure, a material with not only very good optical and thermal properties, but also high mechanical resistance is needed. This material is diamond. Today, by using Chemical Vapor Deposition (CVD) techniques is possible to grow layers of diamonds over large areas relatively cheap, and use them as tritium I vacuum barriers inside high power microwave transmission lines. Diamond presents the aforementioned characteristics (highest thermal conductivity and hardness in nature), a very large bandgap (5.5 eV, very high electrical breakdown threshold) and it is almost insensible to the majority of chemical agents. Finally, they resist a burning flame in atmosphere up to approximately $1500{ }^{\circ} \mathrm{C}[3,4]$. Although perfectly suitable for their intended use, the environmental conditions they'll face and the complexity of the future fusion power plants operations require a constant monitoring of the operating parameters of such diamond windows. Failures can lead to damages to the plant and hazards for the workers. The diagnostics devices to be implemented must have a compact, simple and flexible layout, with a rugged design, in order to maximize serviceability and durability in the harsh surroundings of the windows housing. These diagnostics include arc detection, tritium detection, transmitted / reflected microwave power, pressure and the diamond disk temperature. In this paper a first assessment of the various diagnostics to be implemented in a diamond window is given.

\section{Arc Detection}

The most critical diagnostic to be implemented in a diamond window assembly is the arc detection. Arcs may occur in the case of an electrical field build up and their effects are highly destructive for the surroundings. They can lead to the cracking of the diamond windows,

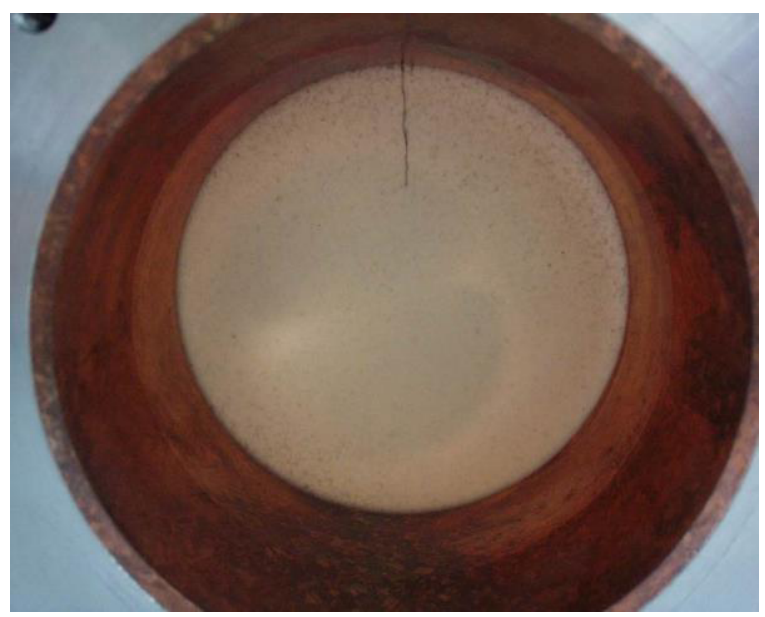

2 Cracked window due to arcing

impairing the transmissivity performances and opening leaks of tritium toward the environment. Arcs will always travel in the direction of the microwave source (the gyrotrons of the facility) and although it is very unlikely that they will be able to reach it, the damage will extend along the discharge path $[11,12]$. It is therefore mandatory to shut down the gyrotrons as soon as the arc event occurs. The concept is to use a fiber optic vacuum feed-through inserted in the outer ring of the copper cuff to bring the light signal to either an Avalanche PhotoDiode (APD) or a PhotoMultiplier Tube (PMT) 
coupled with a PIN diode. The first solution is more compact and rugged and should be the first choice. This configuration allows the detachment of the detector from the cuff, improving accessibility to the device. An interlock system for the gyrotrons triggered by the signal coming from the light sensor is the final component of such a system.

\section{Tritium Detection}

While the insurgence of electrical arcs inside the transmission lines hardly poses any danger for the human workers inside the power plant, tritium represents a serious biological hazard and must be monitored. It's known that the decay radiation spectrum of the beta emitting hydrogen isotope is biased towards low energy electrons (with approx. $18 \mathrm{keV}$ as maximum and $5 \mathrm{keV}$ as average, with the peak emission at approximately 2.5 $\mathrm{keV}$ ) [7] and therefore it's not dangerous as long as it stays outside the human body. Unfortunately, being hydrogen, it readily bind with carbon and hydroxyl radicals, making very easy the absorption by the human body by ingestion, inhalation or through the skin. It also explosively reacts with the oxygen present in the air, which can cause catastrophic damage to both the plant and the human staff. The most straightforward method to detect tritium is to exploit its beta radiation emission. There are various kind of devices that are in principle adapt to this kind of detection, and we can divide them in three categories: gas counters, solid state detectors (diamond detectors) and scintillators. Again, the application in the fusion facilities requires ruggedness, radiation resistance, simplicity and the ability to operate in high temperatures and in the presence of vacuum. Gas detectors (ion chambers, proportional and Geiger counters) detect the radiation by converting it in an electrical current via the generation of electron-ion couples inside a gaseous medium. Depending from the voltage applied between the electrodes, these phenomena may generate an exponential electron avalanche, boosting the sensibility and generating large current signals. Unfortunately they are delicate devices, sensible to environmental changes and with a low sensibility to area ratio, given the low density of their detecting medium. They also require large bias voltage, a potential problem in a high power microwave saturated environment with possible electric arcs. Finally they are sensible to all kind of radiation and can give false positive signals [5].

Diamond detectors work with a similar principle, where the electron-ion pairs are generated inside a crystalline medium instead of a gaseous one. All the characteristics that make diamond a good medium for the windows concur to define diamond detectors performances: they present exceptional resistance to radiation and, as mentioned above, to mechanical stress and chemical attacks; their wide bandgap results in an extremely low dark current, making them suitable, along with the high thermal conductivity, for their use without cooling in a high temperature environment. This is a big plus for this kind of devices, considering also how the temperature oscillates during pulse operation. Finally, their broadband optical transparency means that they are not sensible to

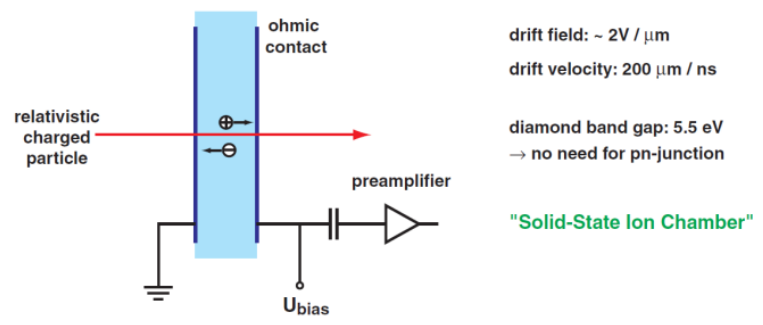

\section{Working principle of a diamond detector}

light pulses, like those that may come from electric arcs. On the other hand the $5.5 \mathrm{eV}$ bandgap cancel all the signal coming from electron with less than that energy, although the percentage of electrons emitted through beta decay with such low energy is extremely low, there will be a considerable amount (about 10\%) of accompanying secondary electrons given by shake-off processes. The detector will still require a bias voltage, in the order of

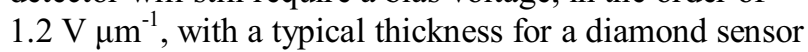
interface around $100-300 \mu \mathrm{m}[3,4,6]$.

Scintillators use a different mechanism, and rely on the coupling with a suitable photodetector. The striking beta particle generates a photon in the scintillating material, that is therefore collected either by an optical amplifier (such as a PMT) or directly by a photodiode. Classic Si photodiodes at zero bias represent the simplest solution, given that such a device doesn't have any amplification stage in the detector itself. Unfortunately several factors reduce the feasibility of such devices in a copper cuff environment. Extremely low energy beta electron will generate fewer photons in the scintillation material, with an even smaller number of them hitting the detector and therefore generating a very low signal [5]. The number of beta particles is quite low, considering that the activity of tritium is measured to be $357 \mathrm{TBq} / \mathrm{g}$ [13], with the total amount of tritium inside a reactor like ITER being roughly 250 grams per day. The temperature range inside the copper cuff stays in a range between 20 and 70 degrees, and even the best photodiodes at those temperatures have dark currents larger than the signal to be detected. They therefore require strong temperature stabilization, given also the nonlinear response of photodiodes over increasing heat. As a final issue, photodiodes typical response spectrum is shifted toward the red / infrared section of the EM spectrum. Suitable scintillation materials are required, and those operating at these wavelengths are not the brightest ones. Photodiodes would be sensible also to arc discharges, therefore a nearly perfect optical isolation is required for the whole detector assembly. It's worth to be noted that these considerations are on a conceptual level only, and definitive conclusions about this topic will be performed after the various diagnostics tests which will be performed for the near future. 
Applying a bias voltage creates a more complex system for sure, but in contrast it increases the sensibility of the detector. It also increases the thickness of the depleted region in the semiconductor, decreasing the capacitance and therefore the electronic noise. Much more sensibility is therefore required, and this can be obtained with the use of APD arrays sensors (or Silicon PhotoMultipliers). In such devices an exponential cascade of impact ionization initiated by the original photogenerated e-h pairs under a bias voltage leads to an avalanche breakdown. In this way even weak optical signals can generate strong electrical signals, capable of overcoming the dark current, reaching even single photon sensitivity. APDs arrays can attain gains in the order of $10^{6}$ with moderate $(50 \mathrm{~V})$ bias voltages. They still require strong temperature stabilization, given the large thermal noise. APDs max efficiency is centered around $550 \mathrm{~nm}$, that it's also the emission wavelength of currently the brightest scintillation material (Ti-doped Cesium Iodate), making the two devices a perfect combination. Visible Light Photon Counters (used in the MICE experiment at RAL and also in the DZero experiment at Fermilab) use As doped Silicon APDs to generate strong gain (approx. $5 \mathrm{x}$ $10^{4}$ ) with a quantum efficiency of 0.9 , with a very small bias (7V). Unfortunately these detectors are also very sensible toward infrared light, and required strong cooling [5]. Everything considered, diamonds detectors are the best choice for this kind of application, with the necessary bias voltage being the only issue so far.

\section{Microwave power leakage measurements}

The third kind of diagnostic is meant to monitor the performance of a diamond window during operation. Transmissivity of the windows is a critical parameter, considering the very high power (in the order of $1 \mathrm{MW}$ or more) microwave beams passing the waveguides. Absorbed radiation will create a hot spot on the window, impairing performances even more. Reflected radiation will return inside the gyrotrons, with catastrophic results. The idea is to add symmetrically up to four horn antennas of appropriate dimensions to the outer ring of the windows housing. Each antenna will be connected to an independent detector channel, and the whole system will provide informations about the power leakage and beam profile that must maintain his Gaussian shape. The two most used detectors for microwave measurements are the superheterodyne and the homodyne. Both come with their cons and pros. Superhet technology is the most widespread in radio detection devices. In this kind of devices the RF signal is amplified in a frequency selective low noise stage, translated into a lower Intermediate Frequency (IF) with additional filtering and amplification and finally downconverted to baseband by mixing or phase discrimination. It is characterized by superior sensitivity and frequency stability, but suffers from problem with interference generated by unwanted input and requires therefore attenuation. The Local
Oscillator (LO) of the system, is also a source of interference and widens the receiver's frequency response as a consequence of its own signal phase modulation [9]. These problems can be bypassed by the use of a homodyne, or direct conversion receiver. In such a device the LO oscillates at the same frequency of the carrier of the incoming radiation. No IF stage is required, being the signal directly fed into the frequency mixer and converted to baseband in a single step. This results in simpler and more compact electronics, with reduced power consumption. All the unwanted beat signals require no additional processing since they are rejected by the use of a low pass filter and fine tuning of the LO. The only flaw is that lacking an IF stage, some functions must be performed at baseband level. In particular, the IF contains an automatic gain control amplifier that must be substituted by a phase lock loop with digital signal processing in order to limit the dynamic range of the output signal [10].

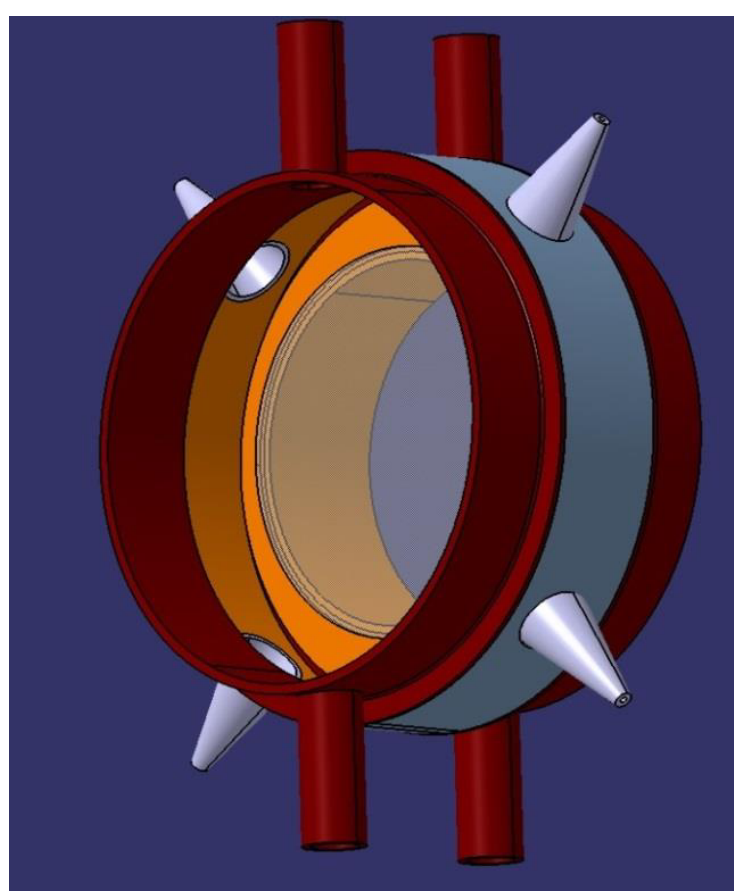

4 Horn antennas for microwave detection

The physical layout of the conceptualized apparatus is composed by up to four horn antennas symmetrically placed around the outer ring of the copper cuff, in order to measure the leakage of microwaves at different points and outline eventual asymmetries in the beam. Simulations on both commercially available and customized dimension antenna models are underway [8], and will be followed by laboratory tests after the realization of the test bench.

\section{Temperature Detection}

Temperature detection is the most straightforward measurement of this group. It can be performed by using thermocouples directly in contact with the outer part of the diamond disk, outside the brazing profile that keeps the window in contact with its copper cuffs. The diamond 
disk extremely high thermal conductivity allows reliable measurements, and the data coming out this diagnostic can be integrated with the microwave measurements for a more precise beam profiling.

\section{Pressure Monitoring}

Vacuum is mandatory inside the reactor and in the wave guides that bring energy to the plasma. It is therefore necessary to monitor the pressure to shut down the machine in case of air infiltration from the outside environment. Recent developments have led to Pirani type microsensors, with dimension in the order of few millimeters, capable of measuring pressures from $10^{3}$ mbar to $10^{-6}$ mbar. These kind of devices can be embedded inside the outer ring of a window assembly, and can be easily substituted in case of failure given their cheap price and fast production.

\section{Conclusions}

This paper provided a preliminary assessment of the diagnostics to be integrated in the diamond windows housing in order to monitor the performances and prevent damages both to the plant and to the workers inside of it. Arc detection and tritium detection are the most critical diagnostics as electrical breakdowns and tritium leakages pose an immediate threat to the surround equipment and personnel. They should be connected to equally fast interlocks, in order to stop operation of the plant as soon as an anomaly is detected. Microwave detection and temperature/pressure control are more important in the operational phase and can be really useful not only to keep the whole system inside the operational parameters, but also to maximize the efficiency of the whole system during the pulse.

"This work, supported by the European Communities under the contract of Association between EURATOM and Karlsruhe Institute for Technology, was carried out within the framework of the European Fusion Development Agreement (GOT4-DIAG WP1). The views and opinions expressed herein do not necessarily reflect those of the European Commission."

\section{References}

[1] ITER Tritium Handbook

[2] ITER Vacuum Handbook

[3] The Charge Collection Properties of CVD Diamond (Behnke, Hüntermeyer ed al.), February 2008

[4] Application of a natural diamond detector for the measurements of relative dose distributions in radiotherapy (S. Vatnisky, H. Jäarvinen), March 1992

[5] Particle detectors and accelerators (K. Nakamura et al.) January 2011

[6] Diamond detectors in particle physics (R.J. Tapper), January 2000

[7] Shake Off mechanism of two electron transition in slow ion-atom collisions (I. Yu Tolstikhina, O.Tolstikhin, H. Tawara) December 1997

[8] Modern Antenna Design Second Edition, T.A. Milligan, 2005

[9] RF Components and Circuits (Joseph J. Carr), 2002
[10] Quad Demodulators Arm Direct-Conversion Receivers, Microwaves and RF (M. Zou, V. Dvorkin, J. Wong)

[11] Performance Of The Arc Detectors Of LHC High Power RF System (D. Valuch et al.) IPAC2011 Proceedings, 2011

[12] Operational Issues at High LH Power Density in JET: Waveguide Conditioning and Arc Detection (M.Goniche et al.) 2012

[13] Radionuclide Safety Data Sheets, Occupational Safety and Environmental Health, University of Michigan (Regents of the University of Michigan) 2010

[14] Pirani type microsensors for pressure measurements from $10^{3} \mathrm{mbar}$ to $10^{-6}$ mbar (M. Grau et al.) DPG 2014 Proceedings, 2014 